Results: 1. Durations of septum after-discharges displayed a moderate increase in relation to time and the frequency distribution patterns were characterized with a trimodal $(6,15$ and $20 \mathrm{c} / \mathrm{sec})$ or unimodal $(3 \mathrm{c} / \mathrm{sec})$ frequency pattern according to the seizure durations respectively.

2) There was no definite correlation between the site of stimulation in the septum and the direction of the blood pressure response. However, the greater pressure elevations appeared more frequently with discharges elicited from the dorsal septal nuclei.

3) The pressor responses occurred with fast frequency discharges and depressor responses with slow frequency discharges. It is suggested that the septum participates in both sympathetic and parasympathetic realms of autonomic activity and that mechanisms exist in control of arterial pressure which are frequency modulated.

4) The septum after-discharge affects blood pressure by implicating hypothalamic elements.

5) The septo-hypothalamic system became an exclusively pressor system after the placement of hypothalamic lesions. However, lesions of the mamillary bodies and its immediate surroundings abolished the blood pressure changes and it is concluded that the septo-hypothalamic blood pressure modulating system is predominantly funneled through these structures.

6) The dorsal hippocampus is the predominant contributor of faster frequencies in septum after-discharge patterns, whereas the ventral hippocampus and amygdala contribute slower frequencies predominantly.

These results rationalize to apply a lesion in these structures to essential hyperor hypotension which might be dependent upon the frequency of after-discharges.

\title{
F-6. DG Potential Changes in Focal Epilepsy and Acute Asphyxia both in Man and Animal
}

\author{
Kazuo MoRI \\ Yhuzo Fujrta*, Hajime Handa and Makoto Kako** \\ *Department of Neurosurgery, Kyoto University Medical School \\ **Department of Neurosurgery, Osaka Red Cross Hospital
}

1) In unanesthetized cats'. laminar DC potential analyses were performed at the epileptic cortex produced by topical application of tungstic acid gel. A pronounced negativity of DC associated with spike in ECoG was created at the superficial layers within the cortex. It was indicated that mass potentials produced at the superficial dendritic aggregates would play a major role for the shifting of surface slow potentials.

2) In 5 cases of cortical epilepsy with depressed fracture, simultaneous recordings of surface DC and ECoG were made at or nearby the focus. As similar to those observed in animals, sporadic spikes in ECoG were associated with negative DC 
the vicinity of the epileptic focus. Conversely, DC went on to positive at an area distant from the focus produced. By observing a direction of DC, we could assertain an active area to which surgical manipuiations (cortical excision etc.) should be undertaken.

3) DC changes during a course of asphyxiation produced by cramping the trachea was also observed in immobilized cats. The asphyxial potential named by Leão followed again by a pronounced slowly on-going negative DC shift which amounted more than $10 \mathrm{mV}$. In corresponding roughly with the stage of negativegoing DC shift, evoked visual cortical response elicited by stimulating the optic tract went out completely and no cases showed a reappearance of ECoG by releasing obstruction of the trachea.

It was concluded that pronounced slowly-going DC shift reflected a critical stage regarding the irreversible loss of a function of the brain.

\title{
F-7. Somatosensory Evoked Responses in Patients with Lesion of Nervous System
}

\author{
Yutaka Hori, Shozaburo Utsumi, Chikayoshi Terada, \\ Atsuo Okazaki, Masatsugu Kobatake and Ryoichi Odani \\ The Second Department of Surgery, Nara Medical Uniuersity
}

There have not been many studies concerning somatosensory evoked responses (SERs) in patients, and the significance of the each component of the SERs has not been satisfactorily clarified on neurophysiological basis. In order to evaluate the abnormal SERs, studies have been made on 182 neurosurgical cases and SERs have been reviewed from the points of clinical application.

SERs were recorded at a point of $2.5 \mathrm{~cm}$ posterior and $7 \mathrm{~cm}$ lateral of the vertex, following the cathodal electrical stimulation, $0.2 \mathrm{msec}$. pulse duration, at a rate of 1 per second, on contralateral median nerve at wrist. A total of 100 or 200 responses were summated. Usually responses were tested at the intensity of motor threshold.

The components of SERs consisted of about seven waves within the first 250 msec. following application of the stimuli.

1) In peripheral nerve lesions, SERs were diminished with impaired proprioceptive sense but not affected with impaired exteroceptive sense as well as motor weakness.

2) SERs were absent or remarkably affected in posterior cervical lesions and less affected in anterior cervical lesions.

3) In cases of diffuse brain stem injuries, the late components of SERs (wave $5,6,7)$ were affected. In the mild cases they were inhibited and showed prolongation of latencies. In moderate disturbances they showed big synchronized waves, and disappeared in advanced disturbances. 